\title{
Epitaxial growth of sexithiophene on mica surfaces
}

\author{
Clemens Simbrunner, ${ }^{1,}$ Gerardo Hernandez-Sosa, ${ }^{1}$ Martin Oehzelt, ${ }^{2}$ Tatjana Djuric, ${ }^{3}$ Ingo Salzmann, ${ }^{4}$ Martin Brinkmann, ${ }^{5}$ \\ Günther Schwabegger, ${ }^{1}$ Irene Watzinger, ${ }^{1}$ Helmut Sitter, ${ }^{1}$ and Roland Resel ${ }^{3}$ \\ ${ }^{1}$ Institute of Semiconductor and Solid State Physics, Johannes Kepler University, Altenbergerstrasse 69, A-4040 Linz, Austria \\ ${ }^{2}$ Institute of Experimental Physics, Johannes Kepler University, Altenbergerstrasse 69, A-4040 Linz, Austria \\ ${ }^{3}$ Institute of Solid State Physics, Graz University of Technology, Petersgasse 16, A-8010 Graz, Austria \\ ${ }^{4}$ Institut für Physik, Humboldt-Universität zu Berlin, Brook-Taylor-Straße 6, D-12489 Berlin, Germany \\ ${ }_{5}^{5}$ Institut Charles Sadron, CNRS - Université de Strasbourg, 23 rue du loess, 67034 Strasbourg, France \\ (Received 1 December 2010; revised manuscript received 9 February 2011; published 24 March 2011)
}

\begin{abstract}
We present a systematic study on the epitaxial growth of $\alpha$-sexithiophene (6T) on two different types of substrates, phlogopite and muscovite mica. The study is based on a structural and morphological analysis using specular $\mathrm{x}$-ray diffraction, $\mathrm{x}$-ray diffraction pole figure technique, transmission electron microscopy, atomic force microscopy, and optical microscopy. It is shown, that $6 \mathrm{~T}$ molecules on mica substrates crystallize in two different configurations, characterized either by the $(\overline{4} 11)$ or $(100)$ contact plane. Whereas the first orientation consists of needle-like structures, the latter one forms an island-like morphology, and both crystal orientations exhibit a well-defined relationship with respect to each other. We demonstrate that this finding can be explained by directed epitaxy of islands along the sidewalls of the needle-like structures.
\end{abstract}

DOI: 10.1103/PhysRevB.83.115443 PACS number(s): 61.46.-w, 81.16.Dn, 61.05.C-, 81.10.-h

\section{INTRODUCTION}

During the last years, the self-assembly of small building blocks such as atoms,,${ }^{1,2}$ molecules, ${ }^{3-6}$ and nanoparticles ${ }^{7,8}$ into macroscopic structures has been intensively studied and recognized as a key technology in the fields of chemistry, biology, physics, and materials science. ${ }^{7}$ In the broad spectrum of available material systems, the epitaxial growth of molecular assemblies on various substrates has been thoroughly investigated and identified as a promising basis for numerous device applications in the field of organic electronics. In this context, the growth of rodlike organic molecules on muscovite mica substrates has been extensively studied ${ }^{9-14}$ and, in particular, phenylenes have been recognized as key materials for a large number of optical applications, e.g., lasing and wave guiding, ${ }^{15}$ due to their advantageous ability to form highly parallel organic nanofibers. To broaden the spectrum of optical applications the phenylene oligomers should be substituted by other rodlike molecules, e.g., acenes or thiophenes, ${ }^{3,13}$ which presupposes a detailed knowledge concerning epitaxial growth.

Here, we report on the epitaxial growth of $\alpha$-sexithiophene (6T) on two different kinds of mica substrates, phlogopite and muscovite mica. It is demonstrated by specular x-ray diffraction, transmission electron microscopy (TEM), and atomic force microscopy (AFM) that 6T tends to crystallize in two distinct kinds of configurations. Whereas island-shaped structures exhibit a (100) contact plane pointing toward quasistanding $6 \mathrm{~T}$ molecules, needle-like structures originate from crystallites, which are characterized by the $(\overline{4} 11)$ contact plane. Furthermore, it is confirmed by x-ray diffraction pole figure (XRD-PF) measurements as well as by TEM that both structures show a well-defined azimuthal orientation, which can be explained by a defined relationship between islandand needle-like structures. In particular, we demonstrate that quasistanding $6 \mathrm{~T}$ molecules perfectly decorate the sidewalls of the observed organic nanoneedles. In addition, the chosen substrates (phlogopite and muscovite mica) exhibit different surface unit cells and therefore allow the studying of their influence on the epitaxial growth of the organic adsorbate. All structural investigations are accompanied by morphological studies using TEM, AFM, and optical microscopy.

\section{EXPERIMENTAL PROCEDURE}

\section{A. Hot-wall epitaxy}

The organic source material 6T (Sigma-Aldrich) has been purified by repeated thermal sublimation circles before being filled into the quarz tube of the hot-wall epitaxy (HWE) reactor. Immediately after cleaving, the muscovite and phlogopite mica substrates (Segliwa $\mathrm{GmbH}$ ) were transferred via a load lock to a HWE evaporation chamber at a base pressure of $9 \times$ $10^{-6}$ mbar. $^{3,13,16}$ In order to reduce the influence of potentially adsorbed species on the surface, before evaporation of the organic compound, a $30 \mathrm{~min}$ in situ preheating procedure at the substrate deposition temperature $\left(90^{\circ} \mathrm{C}\right)$ was performed. Afterwards, the organic source material has been evaporated at $190^{\circ} \mathrm{C}$ for $90 \mathrm{~min}$, heating the wall oven to $220^{\circ} \mathrm{C}$ while the substrate temperature was kept at $90^{\circ} \mathrm{C}$ during the whole deposition process.

\section{B. X-ray diffraction}

Specular X-ray diffraction measurements were performed at the synchrotron radiation source HASYLAB (Hamburg, Germany) using a wavelength of $0.11808 \mathrm{~nm}$. The W1 end station is equipped with a pseudo-z-axis goniometer and a MYTHEN linear detector that spans $\pm 2.3^{\circ}$ in $2 \Theta$. Every specular scan therefore yields a reciprocal space map including a rocking curve at each $q_{Z}$ value.

$\mathrm{X}$-ray diffraction pole figure measurements were performed in Schultz reflective geometry. ${ }^{17}$ Scans were carried out on a Philips X'pert X-ray diffractometer using $\mathrm{Cr} \mathrm{K} \alpha$ radiation and a secondary graphite monochromator. Based on both the observed Bragg peaks of the specular scan and the direction of the poles (net-plane normals) within the pole figures, the 
involved crystallographic phases as well as the alignment of the crystallites with respect to the substrate can be identified.

\section{Transmission electron microscopy}

In addition to the XRD measurements yielding the orientation of 6T crystallites averaged over the sample surface, TEM was used to determine the orientation and the corresponding morphologies of specific domains. The carbon-coated $6 \mathrm{~T}$ film was removed from the muscovite substrate by floating onto a diluted aqueous solution of HF (5\% in weight) and was subsequently recovered onto TEM copper grids. TEM investigations were performed in bright field (BF) and selected area electron diffraction (SAED) modes using a Philips CM 12 electron microscope equipped with a MVIII CCD camera. Calculation of the electron diffraction (ED) patterns was performed with Cerius ${ }^{2}$ software (ACCELYRS).

\section{Morphological investigations}

Optical microscope images have been acquired by a commercially available Nikon Labophot $2 \mathrm{~A}$ microscope in combination with a Nikon Type 115 digital camera. AFM studies of the deposited organic films were performed using a Digital Instruments Dimension 3100 in the tapping mode. The AFM characterization was performed on an area of $30 \times 30 \mu \mathrm{m}^{2}$ with a $\mathrm{SiC}$ tip.

\section{EXPERIMENTAL RESULTS}

In order to understand the epitaxial growth of thiophenes on mica substrates a detailed analysis of the substrate surface morphology, geometry, and composition is essential. Consequently, in a first step geometrical properties of the mica substrate surface unit cells are analyzed and discussed. In a second step a concrete picture about the epitaxial relationship between organic crystallites and the substrate surface is established.

\section{A. Mica substrates}

For our epitaxial growth studies two different types of mica substrates were chosen, namely muscovite and phlogopite mica. Mica crystals - as representatives of phyllosilicatesexhibit a sheetlike structure consisting of tetrahedral and octahedral layers. ${ }^{18}$ One sheet consists of one octahedral layer in between two tetrahedral layers, which are formed by the two building blocks depicted in Fig. 1(a). A partial cation substitution of $\mathrm{Al}^{3+}$ instead of $\mathrm{Si}^{4+}$ atoms in the tetrahedral layers [Fig. 1(a), left panel] generates uncompensated charges within the sheetlike structure. Consequently, the charge deficiency must be balanced by the addition of a cation interlayer provided by potassium atoms. The presence of this interlayer is finally responsible for the perfect cleavage behavior of mica crystals along their $\{001\}$ net planes and the quasi-atomically flat surface after cleavage.

In order to understand the epitaxial growth of molecular crystals on mica surfaces the main differences between muscovite and phlogopite mica are outlined in Figs. 1(b) and 1(c). Whereas muscovite mica belongs to the class of dioctahedral phyllosilicates [Fig. 1(b)], phlogopite can be characterized
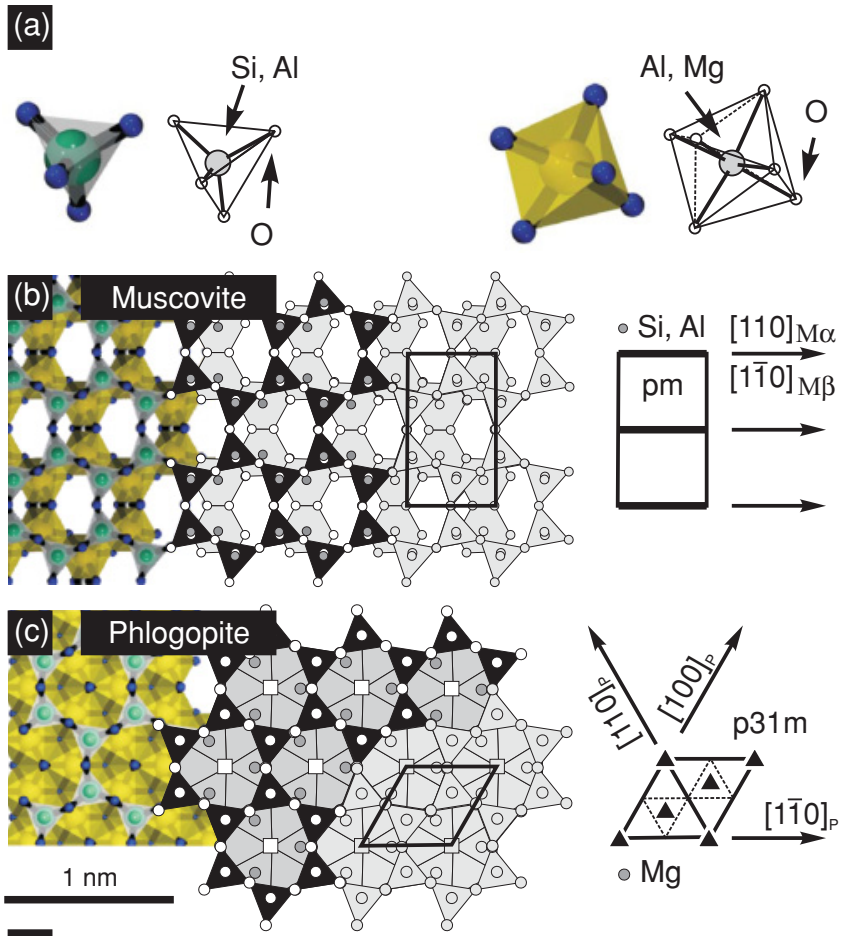

(d)

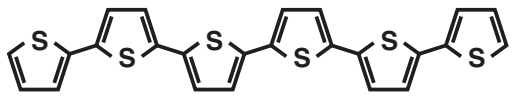

FIG. 1. (Color online) (a) Left: tetrahedral unit built up by $\mathrm{Si}(\mathrm{Al}) \mathrm{O}_{4}$; right: octahedral unit built up by $\mathrm{Al}(\mathrm{Mg}) \mathrm{O}_{6}$. (b) Top view onto a $\{001\}$ surface of a dioctahedral phyllosilicate (muscovite). As indicated by the $\mathrm{pm}$ space group symbol beside, surface symmetry is characterized by parallel aligned mirror axes. (c) Comparable view onto the surface of a trioctahedral phyllosilicate (phlogopite) which can be characterized by three mirror axes as indicated by the $p 31 \mathrm{~m}$ space group symbol. (d) Sexithiophene molecular structure.

as a representative of the trioctahedral group [Figs. 1(c)]. Both groups differ significantly in the morphological and geometrical properties of their substrate surface unit cells. As indicated by gray polygons in Fig. 1(b), vacancies within the octahedral layer of muscovite mica develop, which, in further consequence, causes a distortion within the tetrahedral layer (black triangles) representing the decisive interface for the epitaxial growth. Moreover, the described process is accompanied by a reduction of the substrate surface symmetry and therefore the substrate surface unit cell of muscovite mica can be described by the 2D-space group $\mathrm{pm}$ as indicated in Fig. 1(b). Please note that bold lines represent mirror axes within the figures. In contrast, trioctahedral sheet silicates are characterized by a quasiclosed octahedral layer, which finally leads to less distortion within the tetrahedral sheet. Consequently, phlogopite mica shows a higher degree of symmetry, which can be characterized by the $2 \mathrm{D}$-space group p31m as indicated in Fig. 1(c). Both space groups can be clearly distinguished by the number of mirror symmetry axes (one/three for muscovite/phlogopite) and by their rotational symmetry.

Importantly, the mirror symmetry axes of muscovite mica coincide with parallel surface corrugations caused by lowered oxygen atoms within the tetrahedral layer. Such corrugations 
are not only expected from theoretical considerations but also have been experimentally observed through AFM studies, ${ }^{19}$ drawing a consistent picture concerning the described symmetry of the substrate surface unit cells.

\section{B. Structural and morphological investigations}

The rodlike organic semiconductor $\alpha$-sexithiophene (6T) consists of six linearly linked thiophene rings [indicated in Fig. 1(d)] and is widely used in electronic ${ }^{20}$ and optoelectronic $^{21}$ applications. Consequently, the growth behavior of 6T thin films has been extensively studied on various surfaces. ${ }^{10,12,22-26}$

In a first step structural investigations have been carried out using x-ray diffraction techniques. Specular $\theta / 2 \theta$ scans have been performed to deduce the epitaxial orientation of the $6 \mathrm{~T}$ crystallites relative to the $\{001\}$ net planes of the substrate. Figure 2(a) depicts the results obtained for $6 \mathrm{~T}$ deposited on muscovite mica. An analogous experiment for phlogopite mica leads to an essentially identical diffraction pattern (not shown here). By analyzing the peak positions originating from the organic crystallites, we conclude that the $6 \mathrm{~T}$ molecules crystallize in the known low-temperature phase exhibiting unit cell parameters of $a=44.708 \AA$, $b=7.851 \AA, c=6.029 \AA$, and $\beta=90.76^{\circ} .{ }^{27}$ Two different crystal orientations of $6 \mathrm{~T}$ are present. In particular, we observe the presence of $6 \mathrm{~T}$ crystallites characterized by a parallel alignment of the $\{100\}$ net planes to the mica surface (type $S$ ). Such a crystal orientation is characteristic for quasi-upright
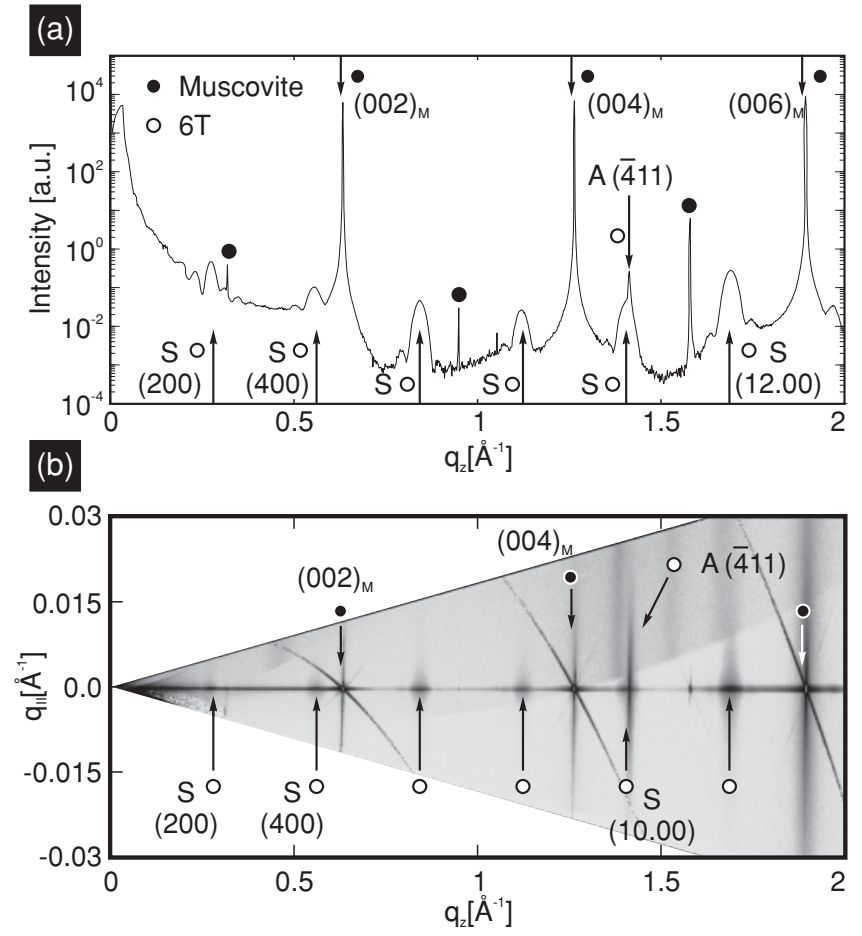

FIG. 2. (a) Specular $\theta / 2 \theta$ scan detected for a $6 \mathrm{~T}$ film grown on a muscovite surface. Solid black circles indicate muscovite mica substrate peaks $(00.2 \mathrm{n})$ and second harmonics. Arrows mark the position of (2h.00) and ( $\overline{4} 11)$ diffraction peaks of $6 \mathrm{~T}$ attributed to crystal orientation $\mathrm{S}$ and A, respectively. (b) $2 \mathrm{D}$ reciprocal space map using a linear detector array. standing 6T molecules on the substrate plane. The series of $2 \mathrm{~h} .00$ peaks for a $6 \mathrm{~T}$ film deposited on muscovite mica is indicated in Fig. 2(a) below the diffraction pattern. In addition, the presence of a second crystal orientation (type A) is indicated by a peak originating from $\{\overline{4} 11\}$ net planes [also indicated in Fig. 2(a)]. This crystal configuration indicates that a portion of the 6T molecules are lying approximately flat on the mica surface. For better visibility, a 2D reciprocal space map is depicted in Fig. 2(b) which clearly resolves the presence of a $(\overline{4} 11)$ peak overlapping with the (10.00) reflection of 6T (type S). No further crystal orientations are observed by XRD and consequently we conclude that these two described orientations are predominantly formed during epitaxial growth.

As both crystal orientations (type $\mathrm{S}$ and A) form significantly different morphologies, XRD results can be nicely correlated with the sample morphology obtained by $\mathrm{AFM}^{12,28}$ for 6T deposited on muscovite [Fig. 3(a)] and phlogopite mica [Fig. 3(b)]. Our AFM analysis clearly reveals these two different crystal morphologies: On the one hand, organic needle structures can be identified and are attributed to crystal orientation of type A. ${ }^{12}$ On the other hand broad islands (type $\mathrm{S}$ ) are visible comprising well-defined terraces between the needles. The step edges of these terraces exhibit a step height of approximately one upright standing molecule (as determined by AFM section analysis), which is in agreement with morphological investigations previously reported in the literature. ${ }^{10,12}$ For better visibility Figs. 3(c) and 3(d) show a zoom for two characteristic morphologies of types A and S, respectively.

Furthermore, XRD-PF measurements have been performed to determine the azimuthal orientation of the observed
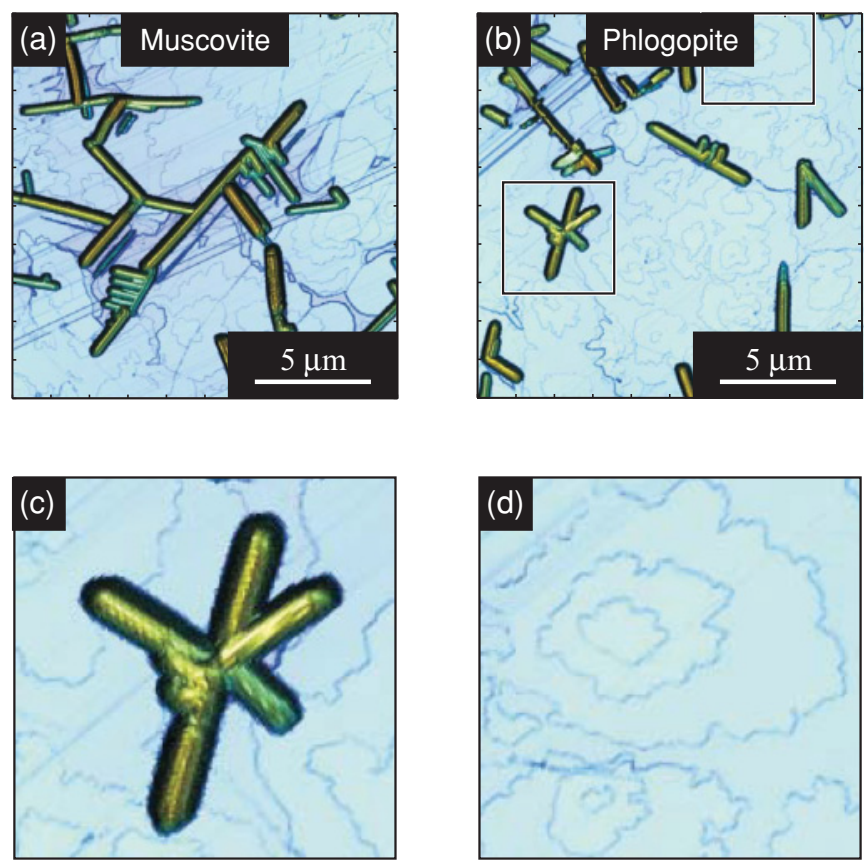

FIG. 3. (Color online) AFM image of 6T deposited on muscovite (a) and phlogopite (b) indicating the presence of needle-like structures (type A) and islands (type S) for both substrates. Magnifications for thin films grown on phlogopite mica are presented in (c)-(d). 
(a)
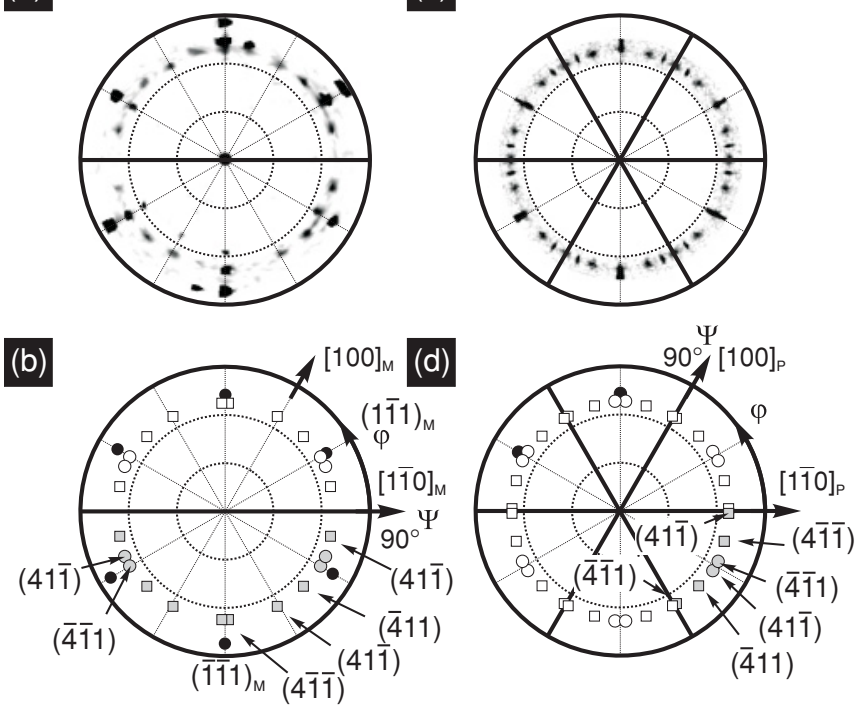

FIG. 4. (a) Pole figure measurement of the $\{\overline{4} 11\}$ net planes of sexithiophene crystals on muscovite (001) surfaces and the corresponding simulation (b). Black filled circles represent reflexes originating from the muscovite mica substrate. Circles/rectangles represent peaks corresponding to $6 \mathrm{~T}$ crystals orientated with their $\{\overline{4} 11\} /\{100\}$ net planes parallel to the substrate surface. White symbols can be constructed from gray symbols due to the symmetry of the substrate surface unit cell. (c)-(d) Analog measurements and simulations for $6 \mathrm{~T}$ grown on phlogopite mica. Bold black lines mark the mirror symmetries of the mica substrate surfaces, one for muscovite and three for phlogopite.

crystallites. A pole figure of the $6 \mathrm{~T}\{\overline{4} 11\}$ net planes is shown in Fig. 4(a). The obtained diffraction peaks can be explained by both type $\mathrm{S}$ as well as type A structures that contribute to the diffraction patterns. As indicated in Fig. 4(a) the obtained pole figure is dominated by a ring of diffraction peaks located at $\Psi=67^{\circ}$. As discrete reflections from the $6 \mathrm{~T}$ adsorbate are visible, both the A-type and the S-type structures have clearly defined azimuthal orientations. A detailed analysis of the experimental data is summarized in Fig. 4(b). The observed diffraction peaks are indexed as follows: rectangles for type $\mathrm{S}$ and circles for type A. Note that the reflections from $\mathrm{S}$ and $\mathrm{A}$ are both found in the same pole figure, since the $\{\overline{4} 11\}$ (type $\mathrm{S}$ ) and $\{41 \overline{1}\}$ (type $\mathrm{A}$ ) are symmetry equivalent (equal lattice spacing). Moreover, the angle between 100 and $\overline{4} 11$ (type S) equals the angle between $\overline{4} 11$ and $41 \overline{1}$ (type A) which causes the reflections of both orientations to occur on a ring within the pole figure. Black solid circles mark positions of substrate peaks and hence provide a unique determination of the azimuthal alignment relative to mica.

A detailed look at the crystal properties of muscovite mica reveals that the bulk unit cell comprises two tetrahedraloctahedral-tetrahedral sheet units. Interestingly, both sheets provide an angular twist of $120^{\circ}$ relative to each other leading to an alternating stacking sequence of type $\alpha \beta \alpha \beta$ along the (001) crystal orientation. Whereas the $\alpha$ planes provide their symmetry axis (and consequently grooved direction) in $[110]_{M}$ direction, the $\beta$ planes show mirror symmetry along $[1 \overline{1} 0]_{M}{ }^{9}$. Therefore it becomes evident that a cleavage step causes the presence of two different surface domains when switching from $\alpha$ to $\beta$ planes and vice versa. ${ }^{9}$ As indicated in Fig. 4(b), the XRD-PF pattern provides mirror symmetry along $[1 \overline{1} 0]_{M}$ demonstrating that the organic crystallites have been grown on a $\beta$ terminated muscovite mica domain. An analogous behavior is observed for $\alpha$ terminated domains, where the organic diffraction peaks are rotated by $120^{\circ}$ and consequently provide mirror symmetry along $[110]_{M}$.

\section{Needle-like structures (crystallites of type A)}

In the following we focus on crystallites of type A, which are characterized by a $\overline{4} 11$ contact plane and consequently consist of quasi-horizontally flat lying $6 \mathrm{~T}$ molecules. It is well known that such a crystal configuration leads to the formation of needle-like structures ${ }^{3,12}$ found by AFM analysis. In the present case the long needle axis (LNA) is defined by the [011] directions of the $6 \mathrm{~T}$ crystal structure and the azimuthal orientations of the LNAs can be directly deduced from XRD$\mathrm{PF}$ analysis since it is known that the (100) planes are lowenergy surfaces which are facets of 6T crystals along the needle axes. A schematic representation of this result is presented in Fig. 5(a) for 6T deposited on muscovite mica. As expected, the geometric alignment of the LNA is perfectly consistent with the surface symmetry of the muscovite mica substrate.

Complementary, the sample morphology has been analyzed by optical microscopy also revealing needle-like structures reaching a length of up to $20 \mu \mathrm{m}$. In order to compare the azimuthal alignment of the LNA with the XRD results, the optical microscope images have been transformed by fast Fourier transformation (FFT); the results are depicted in Fig. 5(b). The FFT image of 6T on muscovite mica is dominated by four radial lines, each representing a

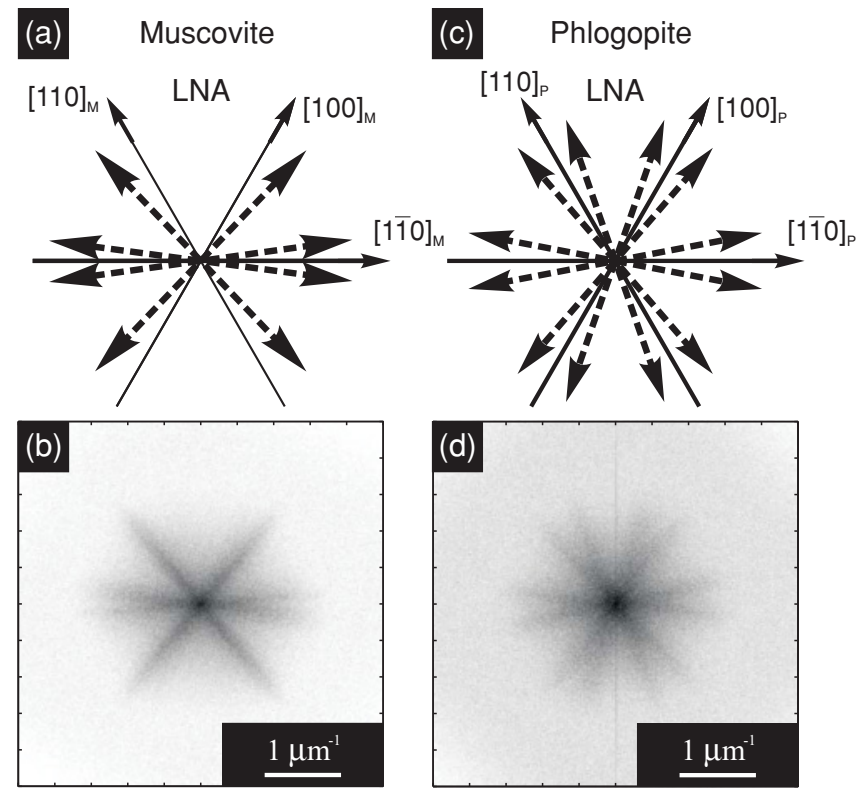

FIG. 5. (a) Azimuthal alignment of the long needle axis (LNA) of $6 \mathrm{~T}$ crystallites orientated with their $\{\overline{4} 11\}$ net planes parallel to the muscovite mica (001) substrate surface. (b) Analog representations for phlogopite mica substrates. (c)-(d) FFT analysis of optical microscope images, taken for 6T crystallites deposited on muscovite mica (c) and phlogopite mica (d). 
specific LNA and it can consequently be directly compared with the XRD results depicted above. It is worth emphasizing that we found perfect agreement between both experimental techniques, concerning not only the quantity of LNAs but also their azimuthal orientation.

Analog investigations have been performed for 6T thin films deposited on phlogopite mica substrates. At first glance the deduced azimuthal configuration appears to be similar to the case of $6 \mathrm{~T}$ on muscovite mica. In particular, the azimuthal alignment of the four needle orientations determined for muscovite mica is again observed. As expected by geometrical considerations concerning the substrate surface geometry, 6T crystallites follow the threefold symmetry of the phlogopite mica substrate and, consequently, further LNAs are present now, as indicated in Fig. 5(c). Again, a direct comparison with optical microscope images can be provided by FFT analysis, which is depicted in Fig. 5(d). Again, a perfect consistence between both experimental techniques is found, which underlines that the predominantly formed needlelike $6 \mathrm{~T}$ crystallites can be entirely described by the $\overline{4} 11$ contact plane.

Based on the combination of our XRD-PF measurements with the known molecular packing within the 6T low-temperature phase, ${ }^{27}$ the azimuthal orientation of $6 \mathrm{~T}$ molecules can now be analyzed in detail. As indicated in Fig. 6(a) for muscovite mica, the long molecular axes (LMAs) of $6 \mathrm{~T}$ are aligned approximately along the $[100]_{M}$ and the $[110]_{M}$ directions of muscovite mica. A real-space model of two 6T crystallites, which correspond to the molecular orientations indicated by red arrows in Fig. 6(a), is

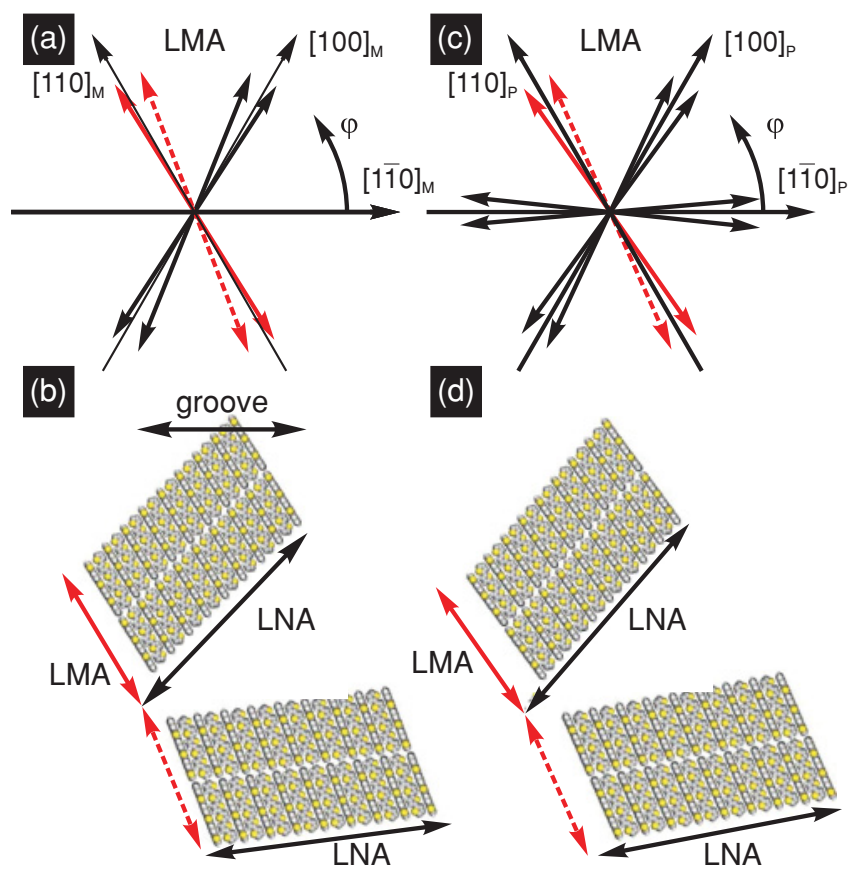

FIG. 6. (Color online) (a) Orientation of the long molecular axes (LMA) deduced from XRD pole figure measurements for $6 \mathrm{~T}$ deposited on muscovite mica. (b) Sketched top view onto 6T crystallites (type A). Note that only two crystallites have been exemplarily selected for the representation. (c)-(d) Analog representations for phlogopite mica substrates. indicated in Fig. 6(b). Both crystallites are characterized by a quasiparallel molecular alignment, whereas their LNAs significantly differ concerning their azimuthal orientations because of the different stacking of $6 \mathrm{~T}$ molecules. We explain this fact as due to the formation of two different crystal types, pointing either with their $(\overline{4} 11)$ or $(4 \overline{11})$ parallel to the substrate. As discussed in more detail elsewhere, ${ }^{29}$ the observed epitaxial relationship is explained by the dynamics of the crystal growth process: In the first growth stage 6T molecules are adsorbed on the muscovite mica surface in a preferred azimuthal orientation, i.e., approximately along the $[100]_{M}$ and the $[110]_{M}$ (in the case of a $\beta$ terminated substrate). The fact that two energetic equivalent molecular orientations exist originates from the substrate surface mirror axis. Upon subsequent growth 6T crystallites are formed and it has been demonstrated by lattice match ${ }^{9}$ and forcefield calculations ${ }^{29}$ that the generated molecular assemblies rearrange and consequently show slightly different molecular orientations.

An analogous behavior is observed for 6T crystallites deposited on phlogopite mica substrates. Again, the LMAs of different organic crystallites may show a slight splitting as indicated in Fig. 6(c). In contrast to 6T grown on muscovite mica, here three preferred molecular adsorption sites are observed, which is explained by the threefold symmetry of the substrate surface. Again, a real-space model is derived and is depicted in Fig. 6(d) for two crystallites corresponding to the LMAs marked by red arrows.

\section{Island-like structures (crystallites of type $S$ )}

As found in the AFM micrographs, in addition to needlelike structures, 6T forms broad island-like structures both on muscovite and phlogopite mica, which correspond to quasistanding 6T molecules grown in the 6T low-temperature crystal phase forming a (100) contact plane to the substrate. The topography of a representative region of the sample showing this specific morphology is depicted in Fig. 7(a). As indicated by different shades of gray (corresponding to height levels) molecular islands are observed between the organic needles discussed above. These structures consist of quasiflat terraces with well-defined and sharp steps, which is demonstrated by the section analysis depicted in Fig. 7(b). This profile was extracted from the path marked by a black line in Fig. 7(a). As indicated by the labels (A-C) each step can be clearly attributed to a gray level in the AFM image and consequently can be seen as representative for equishaded areas. In order to deduce the mean step height of the terraces a height histogram has been derived and is plotted in Fig. 7(c). Three sharp peaks are observed and separated by approximately $2.3 \mathrm{~nm}$, which equals the length of standing 6T molecules with the error margin $(2.24 \mathrm{~nm})$.

To strengthen the XRD and AFM experiments, TEM measurement were performed. In Fig. 8(a) a representative SAED pattern of an area consisting of crystallites of type A as well as of type $S$ is presented. The observed diffraction spots are indexed according to the pole figures (compare Fig. 4): Rectangles are used for type-S and circles for type-A orientation. The defocused SAED image in Fig. 8(b) shows the associated morphologies. The simulated SAED pattern 

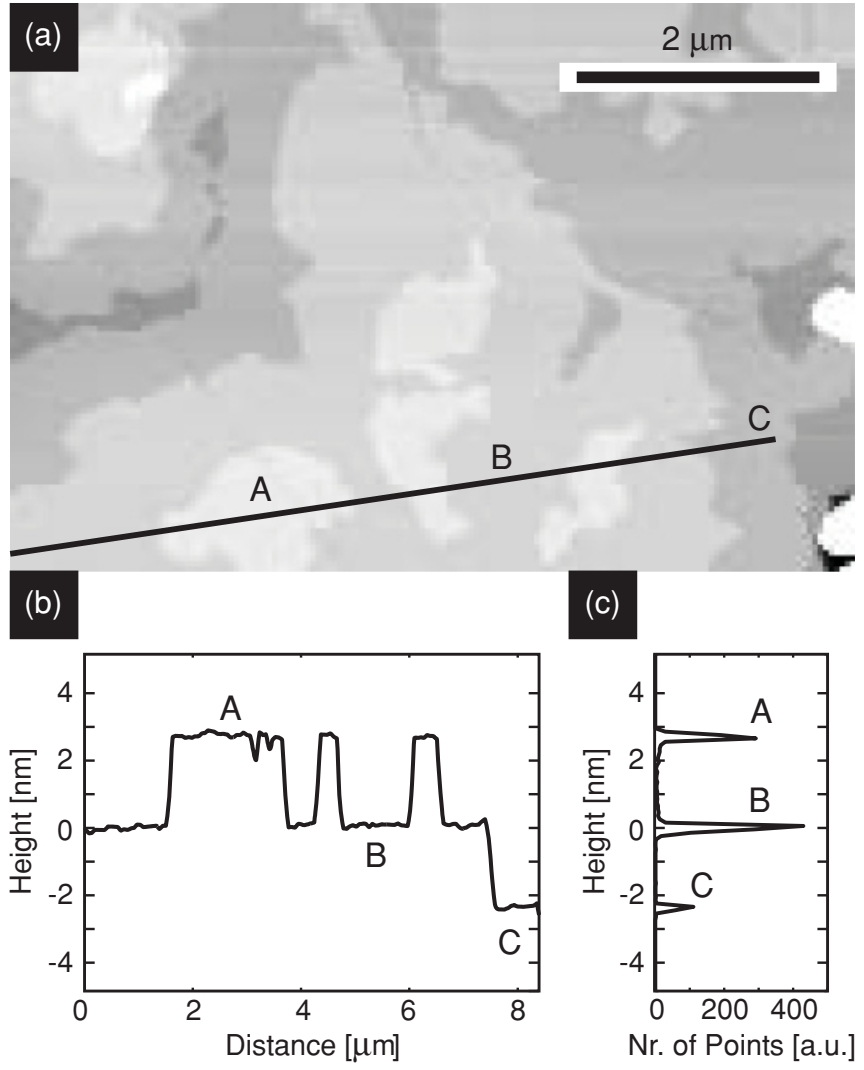

FIG. 7. (a) AFM image showing a representative area providing island-like morphology. (b) Height profile along the direction which is marked by a line in (a). A nicely quantized shape is observed originating from monomolecular 6T steps. (c) Height histogram deduced from the height profile, revealing a terrace step height of approximately $2.3 \mathrm{~nm}$ which nicely corresponds to standing $6 \mathrm{~T}$ molecules.

for the S-type orientation corresponds to the [100] zone axis [Fig. 8(c)] while the simulated pattern for the A-type orientation shown in Fig. 8(d) is corresponding to the $[1 \overline{8} \overline{14}]$ zone axis. TEM offers the unique possibility to link reciprocal to real-space images of the sample. In this way, the assignment of the needle-like morphology to $6 \mathrm{~T}$ crystallites with a $(\overline{4} 11)$ contact plane and that of the island-like morphology to 6T crystallites with a (100) contact plane are ascertained. Moreover, TEM shows that these needle-like structures correspond to edge-on lamellar crystals with a variable height giving rise to different contrasts in the $\mathrm{BF}$ images. Despite the presence of distinct and manifold azimuthal orientations of the 6T crystallites as observed by XRD-PF (Fig. 4) the SAED pattern in Fig. 8(a) further substantiates the proposed arrangement of $6 \mathrm{~T}$ molecules in well-defined single crystalline domains. While for S-type crystallites large and well-ordered domains are observed, crystallites of type A often exhibit slightly tilted orientations. Hence, depending on the specific tilt of the domains different reflexes of the $(\mathrm{h} \overline{2} 1)$ and $(\overline{h 1} 1)$ series are measured [see Fig. 8(d)]. Therefore, the observed diffraction patterns are often asymmetric and less well defined.

In summary, besides a well-defined orientation of standing $6 \mathrm{~T}$ molecules with respect to the substrate normal, XRD-PF
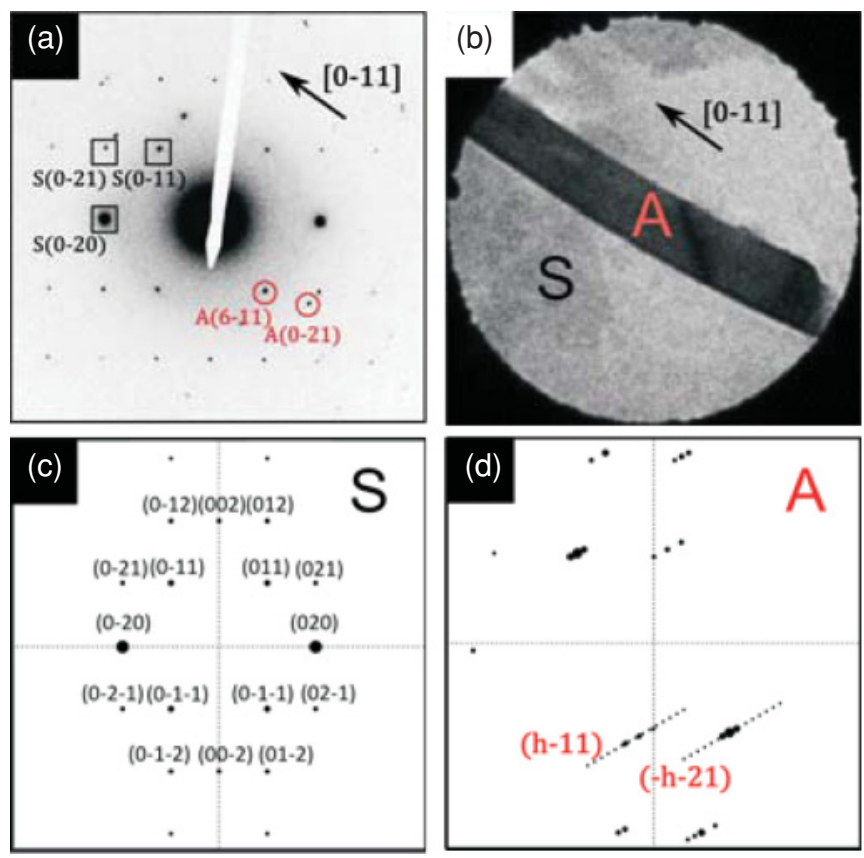

FIG. 8. (Color online) (a) Selected area electron diffraction (SAED) pattern of 6T deposited on muscovite mica. The diffraction spots can be assigned to the typical crystal orientations of the needlelike structures (type A, circles) and of the islands (type S, squares). (b) The defocused SAED image of the corresponding morphologies. Panels (c) and (d) show the simulated electron diffraction patterns of these two orientations which correspond to the zone axes [100] and $\left[\begin{array}{lll}1 & \overline{8} & \overline{14}\end{array}\right]$, respectively.

measurements as well as TEM investigations reveal a clear azimuthal order of the S-type crystallites reflected in a distinct epitaxial relation to the substrate. While the first finding is often observed for epitaxially grown organic thin films, ${ }^{30-33}$ the latter one is a strikingly uncommon finding. ${ }^{28,34,35}$ In order to understand this surprising observation, an in-depth analysis concerning the relationship between crystals of type $S$ and A has been performed. The relationship between the two domains contributing to the SAED pattern shown in Fig. 8(a) reveals that the [011] direction of the S-type crystallites coincides with the [011] direction of the A-type crystallites. The XRD-PF measurements confirm that this is also true averaged over the entire sample: A-type and S-type crystallites are always characterized by a common [01ㅣ direction, which demonstrates the presence of an epitaxial relationship between island-shaped and needle-shaped structures. In addition, their common direction coincides with the long needle axis (LNA) of A-type crystallites, which is easily interpreted when comparing the [01ㅣ direction in the SAED pattern with the orientation of the LNA in the defocused SAED image [Fig. 8(b)].

In order to visualize this phenomenon in more detail the discussed configuration is sketched in Fig. 9 as a realspace model of two adjacent crystallites. Two remarkable observations can be deduced from the presented picture. First, the parallel alignment of the [01ㅣ directions not only points toward an azimuthal relationship but also implies the same periodicity along this direction. Second, it can be rationalized by geometrical considerations that the tilt angle 


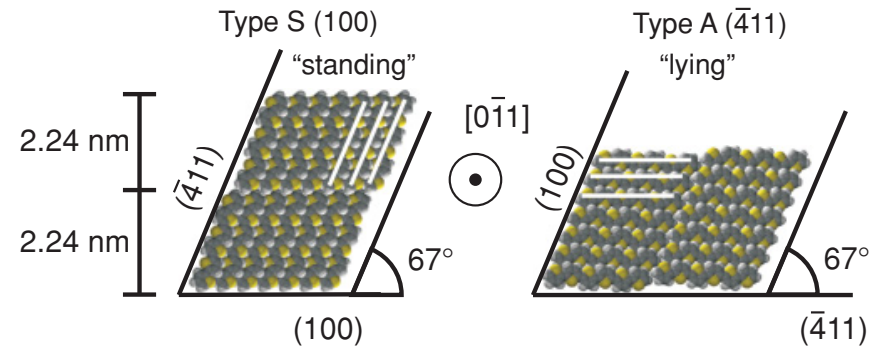

FIG. 9. (Color online) Side view of a real-space model showing the alignment of 6T molecules for the two crystal orientations (left panel: S; right panel: A). The sketch represents a view along the [011] direction of both $6 \mathrm{~T}$ crystallites. White lines mark the alignment of long molecular axes.

$\left(67^{\circ}\right)$ of the molecules within crystals of type $S$ perfectly fits to the inclination of the (100) low index plane of type A. Consequently, it is most likely that the nucleation of the islands consisting of standing molecules takes place at the sidewalls of already existing needles, which also explains the azimuthal orientation and azimuthal relationship between them. Such epitaxial alignment which is not based on the symmetries of the substrate but on the angular match between the facets of the growing organic crystallites is called "ledge-directed epitaxy." 36 This model is further supported by morphological growth studies reporting island nucleation at already grown fibers. ${ }^{10,12,28}$

\section{CONCLUSION}

In summary, we report the epitaxial growth of 6T on two kinds of mica substrates by extensive structural and morphological investigations. Whereas muscovite mica shows lower substrate surface symmetry, phlogopite mica is characterized by a threefold symmetry. We show by $\mathrm{x}$-ray pole figure measurements and FFT analysis of optical microscope images that the geometry of the substrate surface is perfectly reflected in the growth behavior of crystalline organic thin films which were prepared by hot-wall epitaxy. XRD, TEM, and AFM measurements reveal the presence of two different crystallographic orientations. Whereas needle-like structures are characterized by $6 \mathrm{~T}$ crystals with a $(\overline{4} 11)$ contact plane, island-shaped structures are formed by quasistanding molecules with a (100) contact plane. Most strikingly, both types of crystallites show a distinct azimuthal relationship relative to the substrates, which we explain solely by geometrical considerations. The tilt of standing $6 \mathrm{~T}$ molecules perfectly fits the inclination of the sidewall of the organic needle-like structures.

\section{ACKNOWLEDGMENTS}

This work has been financially supported by the Austrian Science Fund (FWF), Projects No. NFN-S9706 and No. NFN-S9708. I.S. acknowledges financial support by the DFG. We thank W. Caliebe (HASYLAB, Hamburg, Germany) for experimental support. *clemens.simbrunner@jku.at

${ }^{1}$ J. V. Barth, G. Costantini, and K. Kern, Nature (London) 437, 671 (2005).

${ }^{2}$ O. Fruchart, M. Klaua, J. Barthel, and J. Kirschner, Phys. Rev. Lett. 83, 2769 (1999).

${ }^{3}$ C. Simbrunner, F. Quochi, G. Hernandez-Sosa, M. Oehzelt, R. Resel, G. Hesser, M. Arndt, M. Saba, A. Mura, G. Bongiovanni, and H. Sitter, ACS Nano 4, 6244 (2010).

${ }^{4}$ F. Balzer and H.-G. Rubahn, Adv. Funct. Mater. 15, 17 (2005).

${ }^{5}$ D.-M. Smilgies, N. Boudet, and H. Yanagi, Appl. Surf. Sci. 1, 24 (2002).

${ }^{6}$ T. Yokoyama, S. Yokoyama, T. Kamikado, Y. Okuno, and S. Mashiko, Nature (London) 413, 619 (2001).

${ }^{7}$ E. V. Shevchenko, D. V. Talapin, N. A. Kotov, S. OBrien, and C. B. Murray, Nature (London) 439, 55 (2006).

${ }^{8}$ M. I. Bodnarchuk, M. V. Kovalenko, S. Pichler, G. Fritz-Popovski, G. Hesser, and W. Heiss, ACS Nano 4, 423 (2010).

${ }^{9}$ R. Resel, T. Haber, O. Lengyel, H. Sitter, F. Balzer, and H.-G. Rubahn, Surf. Interface Anal. 41, 764 (2009).

${ }^{10}$ F. Balzer, M. Schiek, H.-G. Rubahn, K. Al Shamery, and A. Lützen, J. Vac. Sci. Technol. B 26, 1619 (2008).

${ }^{11}$ M. Schiek, A. Lützen, K. Al-Shamery, F. Balzer, and H.-G. Rubahn, Cryst. Growth Design 7, 229 (2007).

${ }^{12}$ L. Kankate, F. Balzer, H. Niehus, and H.-G. Rubahn, Thin Solid Films 518, 130 (2009).

${ }^{13}$ G. Hernandez-Sosa, C. Simbrunner, and H. Sitter, Appl. Phys. Lett. 95, 013306 (2009).
${ }^{14}$ A. Andreev, G. Matt, C. Brabec, H. Sitter, D. Badt, H. Seyringer, and N. S. Sariciftci, Adv. Mater. 12, 629 (2000).

${ }^{15}$ F. Quochi, J. Opt. 12, 024003 (2010).

${ }^{16}$ H. Sitter, D. Stifter, and T. N. Manh, J. Cryst. Growth 174, 828 (1997).

${ }^{17}$ L. G. Schultz, J. Appl. Phys. 20, 1030 (1949).

${ }^{18}$ A. Meunier, Clays (Springer, Berlin Heidelberg New York, 2005).

${ }^{19}$ Y. Kuwahara, Phys. Chem. Miner. 28, 1 (2001).

${ }^{20}$ G. Horowitz, F. Garnier, A. Yassar, R. Hajlaoui, and F. Kouki, Adv. Mater. 8, 52 (1996).

${ }^{21}$ G. Horowitz, F. Kouki, P. Valat, P. Delannoy, and J. Roussel, Phys. Rev. B 59, 10651 (1999).

${ }^{22}$ E. D. Como, M. A. Loi, M. Murgia, R. Zamboni, and M. Muccini, J. Am. Chem. Soc. 128, 4277 (2006).

${ }^{23}$ M. A. Loi, E. D. Como, F. Dinelli, M. Murgia, R. Zamboni, F. Biscarini, and M. Muccini, Nature Mater. 4, 81 (2005).

${ }^{24}$ M. Oehzelt, G. Koller, J. Ivanco, S. Berkebile, T. Haber, R. Resel, F. P. Netzer, and M. G. Ramsey, Adv. Mater. 18, 2466 (2006).

${ }^{25}$ G. Koller, S. Berkebile, J. R. Krenn, F. P. Netzer, M. Oehzelt, T. Haber, R. Resel, and M. G. Ramsey, Nano Lett. 6, 1207 (2006).

${ }^{26}$ M. Koini, T. Haber, S. Berkebile, G. Koller, M. Ramsey, R. Resel, and M. Oehzelt, J. Cryst. Growth 311, 1364 (2009).

${ }^{27}$ G. Horowitz, B. Bachet, A. Yassar, P. Lang, F. Demanze, J.-L. Fave, and F. Garnier, Chem. Mater. 7, 1337 (1995).

${ }^{28}$ T. Haber, R. Resel, A. Andreev, M. Oehzelt, D.-M. Smilgies, and H. Sitter, J. Cryst. Growth 312, 333 (2010). 
${ }^{29}$ C. Simbrunner, D. Nabok, G. Hernandez-Sosa, M. Oehzelt, T. Djuric, R. Resel, L. Romaner, P. Puschnig, C. Ambrosch-Draxl, I. Salzmann, G. Schwabegger, I. Watzinger, and H. Sitter, J. Am. Chem. Soc. 133, 3056 (2011).

${ }^{30}$ I. Salzmann, S. Duhm, G. Heimel, J. P. Rabe, N. Koch, M. Oehzelt, Y. Sakamoto, and T. Suzuki, Langmuir 24, 7294 (2008).

${ }^{31}$ G. Hernandez-Sosa, C. Simbrunner, T. Höfler, A. Moser, O. Werzer, B. Kunert, G. Trimmel, W. Kern, R. Resel, and H. Sitter, Org. Electron. 10, 326 (2009).

${ }^{32}$ M. Oehzelt, R. Resel, C. Suess, R. Friedlein, and W. R. Salaneck, J. Chem. Phys. 124, 054711 (2006).
${ }^{33}$ R. Ruiz, B. Nickel, N. Koch, L. C. Feldman, R. F. Haglund, A. Kahn, and G. Scoles, Phys. Rev. B 67, 125406 (2003).

${ }^{34}$ R. Resel, M. Oehzelt, O. Lengyel, T. Haber, T. U. Schülli, A. Thierry, G. Hlawacek, C. Teichert, S. Berkebile, G. Koller, and M. G. Ramsey, Surf. Sci. 600, 4645 (2006).

${ }^{35}$ J. T. Sadowski, T. Nagao, S. Yaginuma, Y. Fujikawa, A. AlMahboob, K. Nakajima, T. Sakurai, G. E. Thayer, and R. M. Tromp, Appl. Phys. Lett. 86, 073109 (2005).

${ }^{36}$ S. J. Bonafede and M. D. Ward, J. Am. Chem. Soc. 117, 7853 (1995). 